\title{
ON DISSIPATION INEQUALITIES AND LINEAR VISCOELASTICITY*
}

\author{
$\mathrm{Br}$
}

\author{
M. E. GURTIN (Brown University) AND I. HERRERA (University of Mexico)
}

1. Introduction. The purpose of this paper is to derive restrictions which must be placed on the relaxation integral law of linear viscoelasticity theory in order that it satisfy certain "thermodynamic" requirements. This integral law, in the usual indicial notation, may be written as ${ }^{1}$

$$
\sigma_{i j}(t)=\int_{0}^{t} G_{i j k l}(t-\tau) \gamma_{k l}^{(1)}(\tau) d \tau \quad(0 \leq t<\infty),
$$

where $\sigma_{i j}, \gamma_{i i}$, and $G_{i j k l}$ are, respectively, the Cartesian components of the stress tensor, the (infinitesimal) strain tensor, and the tensor of relaxation moduli; while $\gamma_{k l}^{(1)}$ is the first time derivative of $\gamma_{k l}$. If the material is isotropic, then (1.1) reduces to the following pair of one-dimensional laws:

$$
\begin{aligned}
& s_{i j}(t)=\int_{0}^{t} G_{1}(t-\tau) e_{i j}^{(1)}(\tau) d \tau, \\
& \sigma_{k k}(t)=\int_{0}^{t} G_{2}(t-\tau) \gamma_{k k}^{(1)}(\tau) d \tau,
\end{aligned}
$$

where $s_{i j}$ and $e_{i j}$ are the deviators

$$
s_{i j}=\sigma_{i i}-\frac{1}{3} \delta_{i j} \sigma_{k k}, \quad e_{i j}=\gamma_{i j}-\frac{1}{3} \delta_{i j} \gamma_{k k} ;
$$

$G_{1}$ is usually referred to as the shear relaxation modulus and $G_{2}$ as the relaxation modulus for pure compression.

Roughly speaking, our thermodynamic assumption is that work must be done to deform a viscoelastic solid from its virgin state, i.e.

$$
\int_{0}^{t} \sigma_{i j}(\tau) \gamma_{i j}^{(1)}(\tau) d \tau \geq 0
$$

for all sufficiently smooth $\gamma_{i j}$ which satisfy $\gamma_{i j}(0)=0$. We call constitutive relations which have this property dissipative. ${ }^{4}$

${ }^{*}$ Received July 20, 1964.

${ }^{1}$ We tacitly assume that the strain tensor vanishes for all negative time.

2This law, with limitation to isotropic materials, was first formulated by Boltzmann [1] and was later generalized to anisotropic materials by V. Volterra [2]. Coleman and Noll [3] have shown that (1.1) provides a valid first order approximation to the nonlinear theory of simple materials whenever the deformation gradient, taken relative to a natural reference configuration corresponding to zero residual stress, is "small" for all time. Further, Gurtin and Sternberg [4] proved that (1.1) is the most general linear stress-strain relation which is continuous, non-retroactive, and translation-invariant.

sSince our constitutive relation is linear, (1.4) is equivalent to Drucker's [5] postulate. (Added in proof: Coleman [6] has recently developed a fully general thermodynamic theory of materials with fading memory. As one of his many results he proves that in isothermal and isentropic processes starting from equilibrium, the inequality (1.4) holds whenever the path is closed, i.e. whenever $\gamma_{i j}(0)=\gamma_{i j}(t)$.)

"This terminology was introduced by König and Meixner [7] in their study of one-dimensional constitutive relations. 
We show in Section 2 that the work done during a sufficiently accelerated or retarded strain history is approximately elastic, the relevant elasticity tensor being $G_{i j k l}(0)$ for the accelerated history, $G_{i j k l}(\infty)$ for the retarded history. We call $G_{i j k l}(0)$ the instantaneous elasticity tensor; $G_{i j k l}(\infty)$ the equilibrium elasticity tensor.

In Section 3, we prove our main result: If the stress-strain law is dissipative, then both the instantaneous elasticity tensor and the equilibrium elasticity tensor are positive semidefinite and symmetric. ${ }^{5}$ This fact, when coupled with previous results [9] concerning wave propagation in viscoelastic solids, implies the following theorem: If the stressstrain law is dissipative, then there exist three mutually perpendicular acoustic axes, each corresponding to a real speed of propagation.

In Section 4, we demonstrate the connection between dissipative stress-strain laws, functions of positive type, and characteristic functions. This connection allows us to utilize several well known results from probability theory. In particular, we find that the tensor of dynamic viscosities ${ }^{6}$

$$
K_{i j k l}(\omega)=\int_{0}^{\infty} \cos \omega t G_{i j k l}(t) d t
$$

is positive semi-definite whenever the stress-strain law is dissipative. We also prove that the dissipativity postulate, when applied to the one-dimensional laws (1.2) and (1.3), implies that $G_{\beta}$ is continuous and $G_{\beta}(0) \geq\left|G_{\beta}(t)\right|(\beta=1,2)$. The latter result was given previously by Gurtin and Sternberg [4]. ${ }^{7}$

In Section 5, we introduce the notion of strong-dissipativity, which is simply the requirement that the work always be non-negative and vanish for all time only when the strain history is identically zero. We then show that a direct consequence of this postulate is the positive definiteness and (trivially) the symmetry of the instantaneous elasticity tensor. These are precisely the conditions used by Edelstein and Gurtin [11] to establish uniqueness for the mixed problem of linearized dynamic viscoelasticity theory.

The one-dimensional relaxation functions $G_{\beta}(\beta=1,2)$ of $(1.2),(1.3)$ are observed experimentally to be positive and monotone decreasing functions of time. In Section 6 we give counterexamples to prove that these properties cannot possibly be deduced from the postulate of dissipativity, or even strong dissipativity.

Finally we remark that the question as to whether dissipativity implies that $G_{i j k l}(t)$ is symmetric ${ }^{8}$ for all time $t \geq 0$ remains open.

Notation. Throughout this paper $\varepsilon^{N}$ denotes an $N$ dimensional Euclidean vector space. The term vector is used exclusively for an element of $\varepsilon^{N}$. Non-underlined lower

${ }^{b} \mathrm{~A}$ fourth-order tensor $c_{i j k l}$ is symmetric if $c_{i j k l}=c_{k l i j}$; positive semi-definite if $c_{i j k l} \gamma_{i j} \gamma_{k l} \geq 0$. (Added in proof: In a recent paper [8], which is based on the thermodynamic theory of [6], Coleman proves that as a consequence of the second law: $G(0)-G(\infty)$ is positive semi-definite and both $G(0)$ and $G(\infty)$ are symmetric. Onat and Shu have shown us an unpublished manuscript in which they prove that the symmetry of $G(0)$ is implied by the work inequality (1.4). Their result, which was arrived at independently, utilizes a completely different method of proof than ours.

${ }^{6} \mathrm{~A}$ thorough discussion of the relation of the dynamic viscosity to the other mechanical properties is given by Coleman and Markovitz [10].

${ }^{7} \mathrm{Pp} .347,349$.

8Theoretical support for this assumption is usually based on an appeal to Onsager's principle. Rogers and Pipkin [12] question the conclusiveness of these arguments and suggest possible experiments to help settle the issue. Gurtin and Sternberg [13] prove that this symmetry requirement is a necessary and sufficient condition for a certain reciprocal theorem to hold. 
case letters $\alpha, \beta, f, g, \cdots$ represent real numbers or real-valued functions. Underlined lower case letters $f, \gamma, \boldsymbol{\delta}, \xi, \cdots$ designate vectors or vector-valued functions. The inner product of two vectors is written $\boldsymbol{\sigma} \cdot \gamma$ and $|\gamma|=(\gamma \cdot \gamma)^{1 / 2}$. Upper case letters $C, F$, $G, \cdots$ stand for linear transformations-or functions whose values are linear transformations-from $\varepsilon^{N}$ into itself. We write $C^{T}$ for the transpose of $C, \operatorname{tr} C$ for the trace of $C$, and $\|C\|=\left(\operatorname{tr} C^{T} C\right)^{1 / 2}$ for the norm of $C$. Then $|C \gamma| \leq\|C\||\gamma|$. We call $C$ symmetric if and only if $C=C^{T}$, positive semi-definite if and only if $\gamma \cdot C_{\gamma} \geq 0$ for every vector $\gamma$, and positive definite if and only if $\gamma \cdot C_{\gamma}>0$ for every vector $\gamma \neq 0$.

By a history we always mean a function defined on $[0, \infty)$.

The word smooth is used as a synonym for continuously differentiable. Given a function $f$ defined on $[0, \infty)$, then $f^{(1)}$ denotes its first derivative, $f^{(2)}$ its second derivative.

2. The generalized relaxation integral law. Accelerated and retarded strain histories. Consider the generalized ${ }^{9}$ relaxation integral law $\mathscr{L}$ defined by

$$
\mathfrak{L}: \quad \boldsymbol{\sigma}(t)=\int_{0}^{t} G(t-s) \gamma^{(1)}(s) d s \quad(0 \leq t<\infty),
$$

where $\boldsymbol{\gamma}$ is the strain history, $\boldsymbol{\sigma}$ the stress history, and $G$ the relaxation function. To avoid repeated smoothness hypotheses we assume once and for all that the function $G$ is defined and piecewise continuous from the right on $[0, \infty)$. Further we introduce the function space $a=\{\gamma \mid \gamma$ is continuous and piecewise smooth on $[0, \infty), \gamma(0)=0\}$, which will be the domain of definition of the stress-strain law $\&$.

For each strain history $\gamma \varepsilon Q$, the work up to time $t$, which we denote by $w_{\gamma}(t)$, is defined by

$$
w_{\gamma}(t)=\int_{0}^{t} \mathbf{o}(s) \cdot \gamma^{(1)}(s) d s \quad(0 \leq t<\infty),
$$

where $\boldsymbol{\sigma}$ is given by (2.1). Hence

$$
w_{\gamma}(t)=\int_{0}^{t} \int_{0}^{s} \gamma^{(1)}(s) \cdot G(s-\tau) \gamma^{(1)}(\tau) d \tau d s \quad(0 \leq t<\infty) .
$$

The first two theorems which we will prove assert that the work done is approximately elastic for very fast, or very slow, strain histories. To make this precise we need the following definition. Given a strain history $\gamma_{\varepsilon} Q$, then $\gamma_{\alpha} \varepsilon Q$ is defined by

$$
\gamma_{\alpha}(t)=\gamma(\alpha t) \quad(0 \leq t<\infty) .
$$

We call $\gamma_{\alpha}$ an accelerated history if $\alpha>1$; a retarded history ${ }^{10}$ if $\alpha<1$.

Theorem 2.1. If $\gamma \varepsilon a$, then

$$
\lim _{\alpha \rightarrow \infty} w_{\gamma_{\alpha}}\left(\frac{t}{\alpha}\right)=\int_{0}^{t} \gamma^{(1)}(s) \cdot G(0) \gamma(s) d s \quad(0 \leq t<\infty) .
$$

Proof. We begin by choosing $t \varepsilon[0, \infty)$ and $\gamma \varepsilon \propto$ arbitrarily and by letting $\boldsymbol{\delta}(\alpha, s)$ denote the stress at time $s$ corresponding to the accelerated history $\gamma_{\alpha}$, i.e.

$$
\boldsymbol{\sigma}(\alpha, s)=\int_{0}^{s} G(s-\lambda) \gamma_{\alpha}^{(1)}(\lambda) d \lambda \quad(0 \leq s<\infty) .
$$

${ }^{9}$ When $\mathrm{N}=6 \mathscr{L}$ represents $(1.1), \mathcal{E}^{6}$ being the six dimensional space of symmetric second order tensors. On the other hand, if $\mathrm{N}=1$, then $\mathscr{L}$ characterizes either (1.2) or (1.3).

${ }^{10}$ The notion of a retarded history is due to Coleman and Noll [14]. 
Clearly

$$
\gamma_{\alpha}^{(1)}(s)=\alpha \gamma^{(1)}(\alpha s) \quad(0 \leq s<\infty)
$$

and hence

$$
\begin{gathered}
w_{\gamma_{\alpha}}\left(\frac{t}{\alpha}\right)=\int_{0}^{t / \alpha} \mathbf{\sigma}(\alpha, s) \cdot \gamma_{\alpha}^{(1)}(s) d s=\int_{0}^{t} \boldsymbol{\sigma}\left(\alpha, \frac{s}{\alpha}\right) \cdot \gamma^{(1)}(s) d s, \\
\boldsymbol{\delta}\left(\alpha, \frac{s}{\alpha}\right)=\int_{0}^{s} G\left(\frac{s}{\alpha}-\frac{\lambda}{\alpha}\right) \gamma^{(1)}(\lambda) d \lambda \quad(0 \leq s<\infty) .
\end{gathered}
$$

Thus, since $G, \gamma$, and $\gamma^{(1)}$ are bounded on $[0, t]$, and since $G$ is continuous from the right at the origin, Lebesgue's dominated convergence theorem implies

$$
\begin{gathered}
\lim _{\alpha \rightarrow \infty} \delta\left(\alpha, \frac{s}{\alpha}\right)=\int_{0}^{s} G(0) \gamma^{(1)}(\lambda) d \lambda=G(0) \gamma(s) \quad(0 \leq s \leq t) \\
\lim _{\alpha \rightarrow \infty} w_{\gamma_{\alpha}}\left(\frac{t}{\alpha}\right)=\int_{0}^{t} \gamma^{(1)}(s) \cdot G(0) \gamma(s) d s .
\end{gathered}
$$

This completes the proof.

TheOREM 2.2. If $G(\infty)=\lim _{t \rightarrow \infty} G(t)$ exists, then for $\gamma \varepsilon a$

$$
\lim _{\alpha \rightarrow 0} w_{\gamma_{\alpha}}\left(\frac{t}{\alpha}\right)=\int_{0}^{t} \gamma^{(1)}(s) \cdot G(\infty) \gamma(s) d s \quad(0 \leq t<\infty)
$$

Proof. As in the proof of the last theorem we choose $t \varepsilon[0, \infty)$ and $\gamma \varepsilon$ a arbitrarily and let $\delta(\alpha, s)$ be given by (2.5). Then, clearly, (2.7) is satisfied. Thus, if we again make use of Lebesgue's theorem and the properties ${ }^{11}$ of $G$ and $\gamma$, we find that

$$
\begin{array}{ll}
\lim _{\alpha \rightarrow 0} \delta\left(\alpha, \frac{s}{\alpha}\right)=\int_{0}^{s} G(\infty) \gamma^{(1)}(\lambda) d \lambda=G(\infty) \gamma(s) \quad(0 \leq s \leq t) \\
\lim _{\alpha \rightarrow 0} w_{\gamma_{\alpha}}\left(\frac{t}{\alpha}\right)=\int_{0}^{t} \gamma^{(1)}(s) \cdot G(\infty) \gamma(s) d s .
\end{array}
$$

The proof is now complete.

Theorems 2.1 and 2.2 help to motivate the following terminology: We call $G(0)$ the instantaneous elasticity tensor; $G(\infty)$ (if it exists) the equilibrium elasticity tensor.

3. Dissipative stress-strain laws. In this section we will show that if the work done (from the unstrained state) is always non-negative, then the instantaneous and equilibrium elasticity tensors are symmetric and positive semi-definite.

We say that $\mathscr{L}$ is dissipative if and only if

$$
w_{r}(t) \geq 0 \quad(0 \leq t<\infty)
$$

for every $\gamma \varepsilon Q$.

ThEOREM 3.1. If $\&$ is dissipative, then $G(0)$ is symmetric and positive semi-definite.

Proof. Theorem 2.1 and the dissipativity of $\&$ imply

$$
\int_{0}^{t} \gamma^{(1)}(s) \cdot C \gamma(s) d s \geq 0 \quad(0 \leq t<\infty) \text { for every } \gamma \varepsilon Q
$$

"Notice that $G$ is bounded on $[0, \infty)$, since it is piecewise continuous and $G(\infty)$ exists. 
where

$$
C=G(0) .
$$

Let $\Gamma$ denote a piecewise smooth curve in $\varepsilon^{N}$ and let $\int_{\Gamma} C$ stand for the integral of the linear transformation $C$ over $\Gamma$; i.e. if $\omega(s)(0 \leq s \leq t)$ parametrizes $\Gamma$, then $\int_{\mathrm{r}} C=\int_{0}^{t} \omega^{(1)}(s) \cdot C \omega(s) d s$. With this notation (3.1) implies $\int_{\mathrm{r}} C \geq 0$ provided the initial point of $\Gamma$ is the origin. Let the terminal point of $\Gamma$ also be the origin. Then $\int_{-\mathrm{r}} C \geq 0$. On the other hand $\int_{-\Gamma} C=-\int_{\Gamma} C \leq 0$. Thus $\int_{\Gamma} C=0$ whenever $\Gamma$ is closed and passes through the origin. Consequently, $\int_{\Gamma} C=0$ provided only that $\Gamma$ is closed. Hence $C$ is the gradient of a scalar function $\varphi$ on $\varepsilon^{N}$, i.e.

$$
C \xi=\nabla \varphi(\xi)
$$

for every $\xi \varepsilon \varepsilon^{N}$, where $\nabla$ is the gradient in $\varepsilon^{N}$. Therefore $C$ must be symmetric. Moreover, (3.1) and the symmetry of $C$ imply that $C$ is also positive semi-definite. The proof is now complete.

Theorem 3.2. If $\mathcal{L}$ is dissipative, then $G(\infty)$, provided it exists, is symmetric and positive semi-definite.

Proof. Theorem 2.2 and the dissipativity of $\&$ imply that (3.1) is satisfied, where now $C=G(\infty)$. The remainder of the argument is identical to the corresponding portion of the proof of Theorem 3.1.

4. Connection with characteristic functions and functions of positive type. Throughout this section $g$ denotes a real-valued function on $(-\infty, \infty)$. We say that $g$ is of positive type if and only if

$$
\sum_{m=1}^{M} \sum_{n=1}^{M} \mu_{m} \mu_{n} g\left(s_{m}-s_{n}\right) \geq 0
$$

for every integer $M \geq 2$ and every sequence of $2 M$ real numbers $\mu_{1}, \mu_{2}, \cdots, \mu_{M}$, $s_{1}, s_{2}, \cdots, s_{M}$. We call $g$ a characteristic function if and only if there exists a non-decreasing bounded function $\alpha$ on $(-\infty, \infty)$ such that $g$ is the Fourier-Stieltjes transform of $\alpha$, i.e.,

$$
g(t)=\int_{-\infty}^{\infty} \cos \omega t d \alpha(\omega) \quad(-\infty<t<\infty)
$$

The function $\alpha$ is called a distribution function corresponding to $g$. Finally, we say that a real-valued function $f$ on $[0, \infty)$ is of positive type \{is a characteristic function\} if and only if the function $g$ defined by $g(t)=f(|t|)(-\infty<t<\infty)$ is of positive type \{is a characteristic function\}.

For future use we now cite certain well known results concerning characteristic functions and functions of positive type.

LEMma 4.1 (Bochner's Theorem). The function $g$ is of positive type and continuous if and only if it is a characteristic function.

LEMMa 4.2. If $g$ is of positive type, then

$$
g(0) \geq 0, \quad|g(t)| \leq g(0) \quad(-\infty<t<\infty)
$$

If, in addition, $g$ is continuous at the origin, then $g$ is continuous everywhere on $(-\infty, \infty)$. 
Lemma 4.3. If $g$ is a characteristic function which is absolutely integrable on $(-\infty, \infty)$ and if $\alpha$ is a distribution function corresponding to $g$, then $\alpha$ is smooth on $(-\infty, \infty)$ and

$$
\frac{d \alpha(\omega)}{d \omega}=\frac{1}{2 \pi} \int_{-\infty}^{\infty} \cos \omega t g(t) d t \quad(-\infty<\omega<\infty) .
$$

Lemma 4.4. Let $g$ be even on $(-\infty, \infty)$ and piecewise continuous from the right on $[0, \infty)$. Then $g$ is of positive type if and only if

$$
\int_{-t}^{t} \int_{-t}^{t} f(s) f(\tau) g(s-\tau) d s d \tau \geq 0 \quad(0<t<\infty)
$$

for every piecewise continuous function $f$ on $(-\infty, \infty)$.

Proofs of Lemmas 4.1, 4.2, and 4.3 can be found in Loève [15] $]^{12}$. The proof of Lemma 4.4 follows from a slight modification of Mercer's Theorem. ${ }^{13}$

We now establish the connection between the foregoing notions and dissipative stress-strain laws. Henceforth, for every $\xi \varepsilon \varepsilon^{N}, g_{\xi}$ will denote the even function defined by

$$
g_{\xi}(t)=\xi \cdot G(|t|) \xi \quad(-\infty<t<\infty) .
$$

Theorem 4.1. If \& is dissipative, then $g_{\xi}$ is of positive type.

Proof. Given any piecewise continuous function $f$ on $[0, \infty)$ and any vector $\xi \varepsilon \varepsilon^{N}$, the function $\gamma$ defined on $[0, \infty)$ by $\gamma(t)=\xi \int_{0}^{t} f(s) d s$ belongs to $Q$. Thus, if $\&$ is dissipative, then (2.3) and the fact that $g_{\xi}$ is even imply

$$
\int_{0}^{t} \int_{0}^{s} f(s) f(\tau) \xi \cdot G(s-\tau) \xi d s d \tau=\frac{1}{2} \int_{0}^{t} \int_{0}^{t} f(s) f(\tau) g_{\xi}(s-\tau) d s d \tau \geq 0 \quad(0<t<\infty) .
$$

Consequently

$$
\int_{-t}^{t} \int_{-t}^{t} f(s+t) f(\tau+t) g_{\xi}(s-\tau) d s d \tau \geq 0 \quad(0<t<\infty),
$$

for every piecewise continuous function $f$ on $[0, \infty)$ and hence Lemma 4.4 implies that $g_{\xi}$ is of positive type. This completes the proof.

The following result is an immediate consequence of Theorem 4.1, Lemma 4.2, and the properties of $G$.

THEOREM 4.2. If $\&$ is dissipative, then for $(0 \leq t<\infty)$ and every $\xi \varepsilon \varepsilon^{N}$

(i) $\xi \cdot G(t) \xi$ is a continuous function of $t$,

(ii) $|\xi \cdot G(t) \xi| \leq \xi \cdot G(0) \xi$.

This theorem gives us an alternative proof that $G(0)$ is positive semi-definite. Also by (i) and Theorem 3.2 we see that whenever $G(\infty)$ exists:

$$
0 \leq \xi \cdot G(\infty) \xi \leq \xi \cdot G(0) \xi .
$$

For the special case in which the dimension $N$ of $\varepsilon^{N}$ is one, Theorems 4.1 and 4.2, Lemma 4.1, and the above remark imply

Theorem 4.3. If $\&$ is dissipative and $N=1$, then

(i) $G$ is continuous on $[0, \infty)$,

(ii) $|G(t)| \leq G(0)(0<t<\infty)$, 
(iii) $G$ is of positive type,

(iv) $G$ is a characteristic function, and, if $G(\infty)$ exists,

(v) $0 \leq G(\infty) \leq G(0)$.

THEOREM 4.4. If $N=1$ and if $G$ is either a characteristic function or a function of positive type, then $\mathscr{L}$ is dissipative.

Proof. We may assume $G$ is of positive type since, by Lemma 4.1 , it has this property whenever it is a characteristic function. Then under this assumption Lemma 4.4 implies

$$
\int_{-t}^{t} \int_{-t}^{t} f(s+t) f(\tau+t) G(|s-\tau|) d s d \tau \geq 0 \quad(0<t<\infty)
$$

and thus

$$
\int_{0}^{t} \int_{0}^{s} f(s) f(\tau) G(s-\tau) d s d \tau \geq 0 \quad(0<t<\infty) .
$$

Moreover, (4.2) must hold for every piecewise continuous function $f$ on $[0, \infty)$. In particular, if we let $f=\gamma^{(1)}$, where $\gamma \varepsilon Q(\gamma$ is a real-valued function since $N=1)$, then (4.2) and (2.3) imply the dissipativity of $\mathscr{L}$. This completes the proof.

Whenever $G$ is absolutely integrable on $[0, \infty)$ we define the dynamic viscosity ${ }^{\mathbf{1 4}}$ $K$ on $(-\infty, \infty)$ through

$$
K(\omega)=\int_{0}^{\infty} \cos \omega t G(t) d t \quad(-\infty<\omega<\infty)
$$

Theorem 4.5. If $\mathscr{L}$ is dissipative and if $G$ is absolutely integrable on $[0, \infty)$ then $K(\omega)$ is positive semi-definite $(-\infty<\omega<\infty)$.

Proof. It suffices to show that for every $\xi$,

$$
k_{\xi}(\omega)=\xi \cdot K(\omega) \xi \geq 0 \quad(-\infty<\omega<\infty) .
$$

By (4.3)

$$
k_{\xi}(\omega)=\frac{1}{2} \int_{0}^{\infty} \cos \omega t g_{\xi}(t) d t \quad(-\infty<\omega<\infty),
$$

where $g_{\xi}$ is the absolutely integrable function defined by (4.1). Next, it follows from Theorem 4.1, Theorem 4.2, and Lemma 4.1 that $g_{\xi}$ is a characteristic function. Let $\alpha_{\xi}$ be a distribution function corresponding to $g_{\xi}$. Then Lemma 4.3 implies $\alpha_{\xi}$ is smooth and $d / d \omega\left[\alpha_{\xi}(\omega)\right]=1 / \pi k_{\xi}(\omega)$. Thus, since $\alpha_{\xi}$ is a non-decreasing function, $k_{\xi} \geq 0$, and the proof is complete.

If $\mathscr{L}$ represents the one-dimensional law (1.2), then $K$ is the dynamic shear viscosity. It therefore follows as a corollary of Theorem 4.5 that the dynamic shear viscosity is non-negative provided $\mathfrak{L}$ is dissipative.

5. Strongly-dissipative stress-strain laws. We know from the previous section that $G(0)$ is positive semi-definite provided $\mathscr{L}$ is dissipative. The next question we ask is: What postulate assures us that $G(0)$ is positive definite? One is tempted to assume $w_{\xi}(t)>0$ whenever $\gamma$ does not vanish identically on $[0, t]$. However, such a postulate is

\footnotetext{
${ }^{14}$ See Footnote 6.
} 
not satisfied by an elastic solid for which $w_{\gamma}(t)=0$ (at a fixed time $t$ ) whenever $\gamma(t)=\gamma(0)=0$.

The notion of strong-dissipativity - which we now define- seems to us to be physically reasonable. This postulate does not rule out the elastic solid and yields, as a consequence, the positive definiteness of $G(0)$.

We call $\&$ strongly-dissipative if and only if it is dissipative and the only strain history $\gamma \varepsilon Q$ which satisfies

$$
w_{\gamma}(t) \equiv 0 \text { for every } t \geq 0
$$

is the zero history

$$
\gamma(t) \equiv 0 \text { for every } t \geq 0 .
$$

Thus, roughly speaking, the assumption of strong-dissipativity is the requirement that the work always be non-negative and vanish for all time only when the strain history is identically zero.

Theorem 5.1. If $\&$ is strongly-dissipative, then $G(0)$ is symmetric and positive definite.

Proof. By Theorem $3.1 G(0)$ is symmetric and positive semi-definite. Let us assume that $\mathscr{L}$ is strongly dissipative but that $G(0)$ is not positive definite. Then there exists a $\xi \varepsilon \varepsilon^{N}, \xi \neq 0$, such that

$$
\xi \cdot G(0) \xi=0
$$

which, in view of Theorem 4.2, yields

$$
\xi \cdot G(t) \xi=0 \quad(0 \leq t<\infty) .
$$

Thus (2.1) implies (5.1) provided we define $\gamma \varepsilon a$ through $\gamma(t)=t \xi$. However $\gamma$ is not identically zero and we have a contradiction. Hence $G(0)$ is positive definite and the proof is complete.

We now prove a theorem which will be of use to us in the following section.

TheOREM 5.2. If $\&$ is dissipative and if $G$ is twice continuously differentiable on $[0, \infty)$ with $G(0)$ positive definite, then $\&$ is strongly-dissipative.

Proof. Clearly it suffices to show that, under the present hypotheses, (5.1) implies (5.2). The proof of this fact follows almost verbatim the proof of an analogous issue given by Edelstein and Gurtin $[11]^{15}$ which utilizes a method due to Volterra.

We first notice that for $\gamma \varepsilon a(2.1)$ is equivalent to

$$
\boldsymbol{\sigma}(t)=G(0) \gamma(t)+\int_{0}^{t} G^{(1)}(t-s) \gamma(s) d s \quad(0 \leq t<\infty) .
$$

Hence, if we substitute (5.3) into (5.1) and integrate by parts, we find that

$$
\begin{aligned}
\frac{1}{2} \gamma(t) \cdot G(0) \gamma(t)+\int_{0}^{t} \gamma(t) & \cdot G^{(1)}(t-\tau) \gamma(\tau) d \tau-\int_{0}^{t} \gamma(\tau) \cdot G^{(1)}(0) \gamma(\tau) d \tau \\
& -\int_{0}^{t} \int_{0}^{\tau} \gamma(\tau) \cdot G^{(2)}(t-s) \gamma(s) d s d \tau=0 \text { for every } t \geq 0 .
\end{aligned}
$$

Since $G(0)$ is positive definite there $\operatorname{exists}^{16}$ a $\delta>0$ such that $\gamma(t) \cdot G(0) \gamma(t) \geq \delta|\gamma(t)|^{2}$.

${ }^{15}$ Lemma 3, p.11.

${ }^{10}$ Take $\delta$ equal to the smallest eigenvalue of $G(0)$. 
Thus if we let $\omega(t)=|\gamma(t)|$ and

$$
\beta\left(t_{0}\right)=\frac{2}{\delta} \sup _{\left(0 \leq t \leq t_{0}\right)}\left(\left\|G^{(1)}(t)\right\|+\left\|G^{(2)}(t)\right\|\right)
$$

then $\beta\left(t_{0}\right)<+\infty$ and

$$
\begin{aligned}
\omega(t)^{2} \leq \frac{1}{\delta} \gamma(t) \cdot C_{\gamma}(t) \leq \beta\left(t_{0}\right)\left[\int_{0}^{t} \omega(t) \omega(\tau)\right. & d \tau \\
& +\int_{0}^{t} \omega(\tau)^{2} d \tau \\
& \left.+\int_{0}^{t} \int_{0}^{\tau} \omega(\tau) \omega(s) d \tau d s\right] \quad\left(0 \leq t \leq t_{0}\right) .
\end{aligned}
$$

The last integral is equal to $\frac{1}{2}\left[\int_{0}^{t} \omega(\tau) d \tau\right]^{2}$ which, because of the Schwarz inequality, is $\leq t_{0} / 2 \int_{0}^{t} \omega(\tau)^{2} d \tau\left(0 \leq t \leq t_{0}\right)$. Consequently,

$\omega(t)^{2} \leq \beta\left(t_{0}\right)\left[\int_{0}^{t} \omega(t) \omega(\tau) d \tau+\left(1+\frac{t_{0}}{2}\right) \int_{0}^{t} \omega(\tau)^{2} d \tau\right] \quad\left(0 \leq t \leq t_{0}\right)$.

We now prove, by induction, that for every $t \varepsilon\left[0, t_{0}\right]$

$$
\omega(t)^{2} \leq \rho\left(t_{0}\right) \frac{\left[\kappa\left(t_{0}\right) t\right]^{m}}{m !} \quad(m=0,1,2, \cdots \cdots),
$$

where

$$
\begin{aligned}
& \rho\left(t_{0}\right)=\sup _{\left(0 \leq t \leq t_{0}\right)} \omega(t)^{2}, \\
& \kappa\left(t_{0}\right)=\beta\left(t_{0}\right)\left[3+\frac{t_{0}}{2}\right] .
\end{aligned}
$$

This will complete the proof since the right-hand side of (3.5) tends to zero as $m \rightarrow \infty$ and $t_{0}$ is arbitrary. Certainly (5.5) holds for $m=0$. We take as our induction hypothesis that (5.5) is true for $m=r$. Since $\left[\tau^{r / 2} \omega(t)-t^{r / 2} \omega(\tau)\right]^{2} \geq 0$, it follows that

$$
2 \omega(t) \omega(\tau) \leq t^{-r / 2} \omega(t)^{2}+\tau^{-r / 2} \omega(\tau)^{2}
$$

Thus, by the induction hypothesis we have for $t, \tau \varepsilon\left(0, t_{0}\right]$

$$
\omega(t) \omega(\tau) \leq \rho\left(t_{0}\right) \frac{\kappa\left(t_{0}\right)^{r}}{r !} \tau^{r / 2} t^{r / 2} .
$$

If we take $t=0$ in (5.4), we see that $\omega(0)=0$ and hence (5.6) also holds when either $\tau=0$ or $t=0$. The induction hypothesis, together with (5.4) and (5.6), now yields

$$
\omega(t)^{2} \leq \rho\left(t_{0}\right) \frac{\left[\kappa\left(t_{0}\right) t\right]^{r+1}}{(r+1) !} \quad\left(0 \leq t \leq t_{0}\right),
$$

which completes the proof.

6. Counter-examples. The (one-dimensional) relaxation functions encountered in rheology are always positive and monotone decreasing. It is natural to ask whether such a property could possibly follow from the assumption of dissipativity. The next theorem shows that the answer to this question is "no."

TheOREM 6.1. If the dimension $N$ of $\varepsilon^{N}$ is one and if

$$
G(t)=\cos t \quad(0 \leq t<\infty),
$$

then $\mathfrak{L}$ is strongly-dissipative. 
Proof. Since $G(0)=+1$ we conclude from Theorem 5.2 that $\&$ is strongly dissipative provided it is dissipative. By Theorem $4.4 \&$ is dissipative whenever $G$ is a characteristic function, i.e., whenever there exists a non-decreasing bounded function $\alpha$ on $(-\infty, \infty)$ such that

$$
G(t)=\int_{-\infty}^{\infty} \cos \omega t d \alpha(\omega) \quad(0 \leq t<\infty) .
$$

Clearly $\alpha$ defined by

$$
\alpha(\omega)= \begin{cases}0 & (-\infty<\omega<1) \\ 1 & (1 \leq \omega<\infty)\end{cases}
$$

satisfies these conditions and the proof is complete.

The relaxation function $G(t)=\cos t$ of the last theorem has the property that $G(\infty)$ does not exist. In the next theorem we give a counterexample for which $G(\infty)$ exists and equals zero.

Theorem 6.2. If the dimension $N$ of $\mathcal{E}^{N}$ is one and if

$$
G(t)=e^{-t} \cos t \quad(0 \leq t<\infty),
$$

then \& is strongly-dissipative.

Proof. As in the last theorem it suffices to find a distribution function $\alpha$ such that (6.2) holds, provided $G$ is given by (6.3). It may be easily verified (with the aid of a contour integration) that

$$
\alpha(\omega)=\frac{1}{\pi} \int_{0}^{\omega} \frac{\lambda^{2}+2}{\lambda^{4}+4} d \lambda \quad(-\infty<\omega<\infty)
$$

is such a distribution function. This completes the proof.

The form of $G(t)$ in (6.3) was chosen for simplicity. Actually the theorem is valid whenever $a \geq 0$ and

$$
G(t)=e^{-a t} \cos b t \quad(0 \leq t<\infty) .
$$

Acknowledgements. The authors gratefully acknowledge valuable discussions with B. D. Coleman, H. Krieger, and A. C. Pipkin.

This research was supported in part (MEG) by the Office of Naval Research under Contract Nonr 562(25) with Brown University and in part (IH) by the Institutes of Geophysics and Engineering at the University of Mexico.

\section{REFERENCES}

[1] Boltzmann, L.: Zur Theorie der elastischen Nahwirkung. Sitzber. Akad. Wiss. Wien, Math. naturw. K1. 70, 275 (1874)

[2] Volterra, V.: Sulle equazioni integro-diffenziali della teoria dell'elasticita. Att. Real. Accad. Linc. 18, 295 (1909)

[3] Coleman, B. D. and W. Noll: Foundations of linear viscoelasticity. Rev. Mod. Phys. 33, 239 (1961)

[4] Gurtin, M. E. and E. Sternberg: On the linear theory of viscoelasticity. Arch. Rational Mech. Anal. 11, 291 (1962)

[5] Drucker, D.: A definition of a stable inelastic material. J. Appl.. Mech. 26, 101 (1959)

[6] Coleman, B. D.: Thermodynamics of materials with memory. To appear in the Arch. Rational Mech. Anal., 17, 1 (1964) 
[7] König, H. and J. Meixner: Lineare Systeme und lineare Transformationen. Math. Nach. 19, 256 (1958)

[8] Coleman, B. D.: On thermodynamics, strain impulses and viscoelasticity. To appear in the Arch. Rational Mech. Anal., 17, 3 (1964)

[9] Herrera, I. and M. E. Gurtin: A correspondence principle for viscoelastic wave propagation. To appear in the Quart. Appl. Math.

[10] Coleman, B. D. and H. Markovitz: Normal stress effects in second-order fluids. J. Appl. Phys. 35, 1 (1964)

[11] Edelstein, W. and M. E. Gurtin: Uniqueness theorems in the dynamic linear theory of anisotropic viscoelastic solids. To appear in the Arch. Rational Mech. Anal.

[12] Rogers, T. G. and A. C. Pipkin: Asymmetric relaxation and compliance matrices in linear viscoelasticity. ZAMP. 14, 334 (1963)

[13] Gurtin, M. E. and E. Sternberg: A reciprocal theorem in the linear theory of viscoelastic solids. J. Soc. Indust. Appl. Math. 11, 607 (1963)

[14] Coleman, B. D. and W. Noll: An approximation theorem for functionals with applications in continuum mechanics. Arch. Rational Mech. Anal. 5, 355 (1960)

[15] Loève, M.: Probability theory. Van Nostrand, New York (1955)

[16] Widder, D. V.: The Laplace transform. Princeton (1941) 